\title{
Analytical Formulas for the Directivity of General Antenna Arrays.
}

\author{
E. Van Lil, J.-w. De Bleser, A. Van de Capelle \\ Div. ESAT-TELEMIC \\ K.U.Leuven \\ Kasteelpark Arenberg, 10; Bus 2444; B-3001 Heverlee, Belgium \\ Emmanuel.VanLil@ESAT.KULeuven.Be
}

\begin{abstract}
Some formulas for rotationally symmetric patterns, as well as an optimal computation of directivities have already been published. The previous results are generalized in this paper for completely arbitrary element patterns.
\end{abstract}

Antenna Arrays; Directivity; analytical expressions, indoor propagation

\section{INTRODUCTION}

The directivity function, the ratio between the power density in a certain direction and the average power density, is defined in [1] and [2],

$D(\theta, \phi)=\frac{|\vec{F}(\theta, \phi)|^{2}}{\frac{1}{4 \pi} \int_{0}^{2 \pi} \int_{0}^{\pi} \vec{F}(\theta, \phi) \overrightarrow{F^{*}}(\theta, \phi) \sin \theta d \theta d \phi}=\left|\overrightarrow{F^{\prime}}(\theta, \phi)\right|^{2} D$

$\vec{F}(\theta, \phi)$ is the complex far-field pattern (* denotes complex conjugate),

$$
\overrightarrow{F^{\prime}}(\theta, \phi)=\frac{\vec{F}(\theta, \phi)}{F_{M A X}} \text { is the normalised field of the array }
$$

(electric or magnetic) and D is the directivity in the direction of maximum radiation. For an array of $\mathrm{N}$ elements, the far-field pattern can be written as the product of an element pattern and an array factor. The array factor depends on the (complex) excitation coefficients $\left(a_{i}\right)$ and the positions of the elements as well as on the frequency. The maximum value occurs if all amplitudes add up in phase, hence (n=N-1) $f_{M A X}=\sum_{i=0}^{n}\left|a_{i}\right|$. For the most common arrays this occurs within the far-field pattern corresponding with real angles $\theta$ and $\phi$.

\section{COMPUTATIONS}

\section{A. Previous derivations}

Using [3, 8.411.1], we have shown that the denominator of (1) for vertical Hertzian dipoles could be expressed as:

$$
\begin{aligned}
& N_{D}=2 \sum_{i=0}^{n} \sum_{j=i+1}^{n} \operatorname{Re}\left(a_{i} a_{j}^{*}\right)\left[f_{1}\left(k_{0}\left|\overrightarrow{p_{i}}-\overrightarrow{p_{j}}\right|\right)-f_{2}\left(k_{0}\left|\overrightarrow{p_{i}}-\overrightarrow{p_{j}}\right|\right) k_{0}^{2}\left(z_{i}-z_{j}\right)^{2}\right] \\
& \quad+\frac{2}{3} \sum_{i=0}^{n}\left|a_{i}\right|^{2} \\
& \overrightarrow{p_{i}}-\overrightarrow{p_{j}}=\left(\overrightarrow{r_{i}}-\overrightarrow{r_{j}}\right)-\left(\overrightarrow{r_{i}}-\overrightarrow{r_{j}}\right) \cdot \overrightarrow{i_{z}} \vec{i}_{z}
\end{aligned}
$$

$p$, denoting the projection of the array element centres on a plane (in this case the $x-y$ plane). The smooth distance functions $f_{1}$ and $f_{2}$ could be written as spherical Bessel functions as follows:

$$
\begin{aligned}
& f_{1}(x)=j_{0}(x)-\frac{j_{1}(x)}{x} \\
& f_{2}(x)=-\frac{j_{2}(x)}{x^{2}}
\end{aligned}
$$

These have a zero derivative for small intermediate distances between antenna elements and look like Fig. 1:

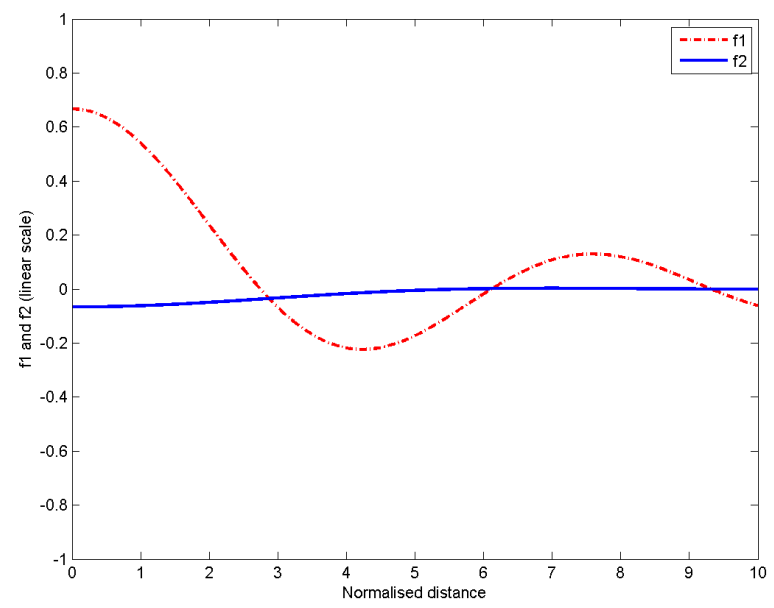

Fig. 1: distance functions for Hertzian dipoles.

For the isotropic radiators the function $f_{l}$ is obviously the known sinc function. Those functions can be expressed in terms of trigonometric functions as follows: 


$$
\begin{aligned}
& f_{1}(x)=\operatorname{sinc} x-\frac{\operatorname{sinc} x-\cos x}{x^{2}} \\
& f_{2}(x)=\frac{x \sin x+3 \cos x-3 \operatorname{sinc} x}{x^{4}}
\end{aligned}
$$

With limits for $\mathrm{d} \rightarrow 0$ equal to:

$$
\begin{aligned}
& \lim _{x \rightarrow 0} f_{1}(x)=2 / 3 \\
& \lim _{x \rightarrow 0} f_{2}(x)=-1 / 15
\end{aligned}
$$

\section{B. Generalisation}

The first generalization concerned patterns that were only dependent of $\theta$. In [5], we found that, for a $\cos ^{\mathrm{m}} \theta$ pattern, it was possible to write the denominator as:

$$
\begin{aligned}
N_{D}= & \sum_{i=0}^{n} \sum_{j=0}^{n} a_{i} a_{j}^{*} \sum_{k=0}^{m} \frac{(-1)^{m-k} m !(2(m-k)) !}{k !(m-k) !^{2}\left(2 k_{0}\left|\overline{p_{i}}-\overline{p_{j}}\right|\right)^{m-k}} \\
& j_{m-k}\left(k_{0}\left|\overline{p_{i}}-\overline{p_{j}}\right|\right)
\end{aligned}
$$

This can be more explicitly written as:

$$
\begin{aligned}
N_{D}= & \sum_{i=0}^{n} \sum_{j=0}^{n} a_{i} a_{j}^{\prime \prime}\left[j_{0}\left(\alpha_{i j}\right)-C_{m}^{1} \frac{j_{1}\left(\alpha_{i j}\right)}{\alpha_{i j}}+3 C_{m}^{2} \frac{j_{2}\left(\alpha_{i j}\right)}{\alpha_{i j}^{2}}-\ldots\right. \\
& \left.+(-1)^{m} 1.3 \ldots(2 m-1) \frac{j_{m}\left(\alpha_{i j}\right)}{\alpha_{i j}^{m}}\right]
\end{aligned}
$$

with $\alpha_{i j}=k_{0}\left|\overline{p_{i}}-\overline{p_{j}}\right|$, only dependent on $\mathrm{i}-\mathrm{j}$ for linear equidistant arrays.

As a special case, for $\mathrm{m}=2$ also a simple formula is obtained:

$$
N_{D}=\sum_{i=0}^{n} \sum_{j=0}^{n} a_{i} a_{j}^{*}\left[j_{0}\left(\alpha_{i j}\right)-\frac{2 j_{1}\left(\alpha_{i j}\right)}{\alpha_{i j}}+\frac{3 j_{2}\left(\alpha_{i j}\right)}{\alpha_{i j}^{2}}\right]
$$

For a linear equidistant array, the directivities can be computed in a fast way, by making use of the power coefficients:

$$
\begin{gathered}
c_{l}=\sum_{i=0}^{n-l} a_{i} a_{i+l}^{*} \\
N_{D}=2 \sum_{l=0}^{n} \operatorname{Re}\left(c_{l} e^{-j l\left(\overline{k_{M A X}}-\overline{k^{\prime} M A X}\right) \cdot \vec{d}}\right) F\left(l k_{o} d\right)+c_{0} F(0)
\end{gathered}
$$

where $F$ is the function of the spherical Bessel functions and $\mathrm{k}_{\text {max }}$ being the direction of the beam steered maximum (the unsteered one being at $\mathrm{k}_{\max }$. For practical applications (corporate feeding of the array) the coefficients vary little with frequency and hence the $c_{l}$ s can be computed in advance. In that case the geometric layout looks like Fig. 2.

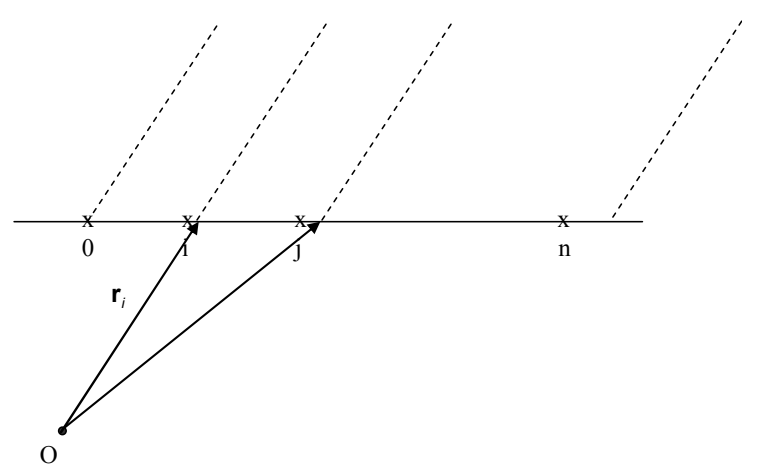

Fig. 2: linear array.

To program this efficiently all $c_{l}$ s are computed in advance in one vector, destroying the excitation coefficients. Until the $(\mathrm{E}(\mathrm{N} / 2)-1)^{\text {th }}(\mathrm{E}$ is the integer just lower than or equal to $\mathrm{N} / 2$ ) calculation of $c_{I}$ the new element is added in the vector after the end of vector a $\left(c_{l}\right.$ is added in $\left.a_{n+1}\right)$ (Fig. 3 a). The total number of memory elements is thus $\mathrm{N}+\mathrm{E}(\mathrm{N} / 2)-1=\mathrm{E}((3 \mathrm{~N}-2) / 2)$. After the $(\mathrm{E}(\mathrm{N} / 2)-1)^{\mathrm{th}}$ coefficient $c_{l}$, the next coefficient $c_{l}$ can be placed directly in element $a_{l}$, because those excitation coefficients are not longer required (Fig. 3 b). At this stage the original excitation coefficients start to be destroyed in the computation. After the buffering the first elements can be copied in place. This means that $a_{n+1}=c_{1}$ is put back at memory location $a_{1}, a_{n+2}$ on $a_{2}$ etc (Fig. $3 \mathrm{c}$ ).

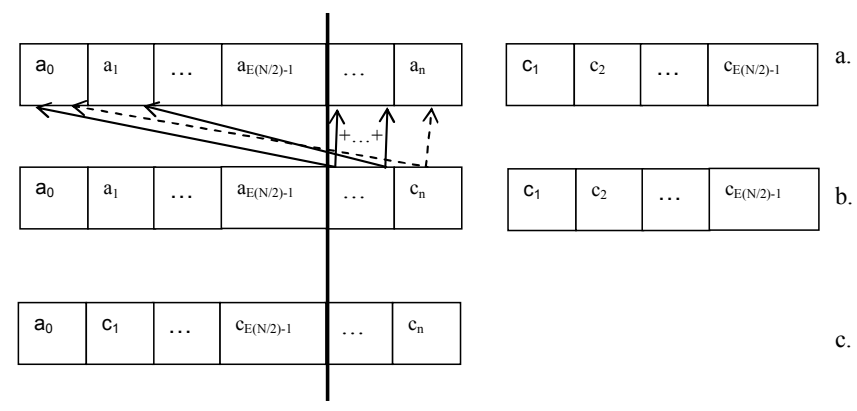

Fig. 3: Efficient computation of the coefficients cl for equidistant arrays

An example for an 8 element uniform array is given in Fig. 4. 


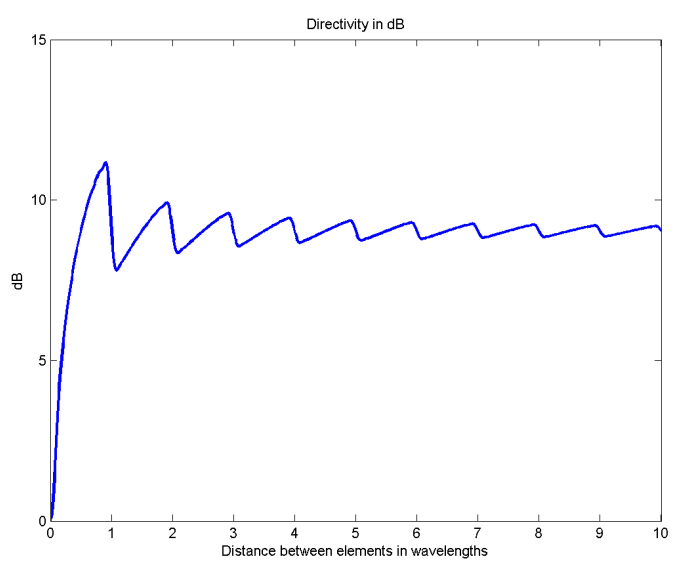

Fig. 4: Efficient computation of the directivity of an 8 element linear array

An example for an 8 element $-20 \mathrm{~dB}$ Chebychev array is given in Fig. 4.

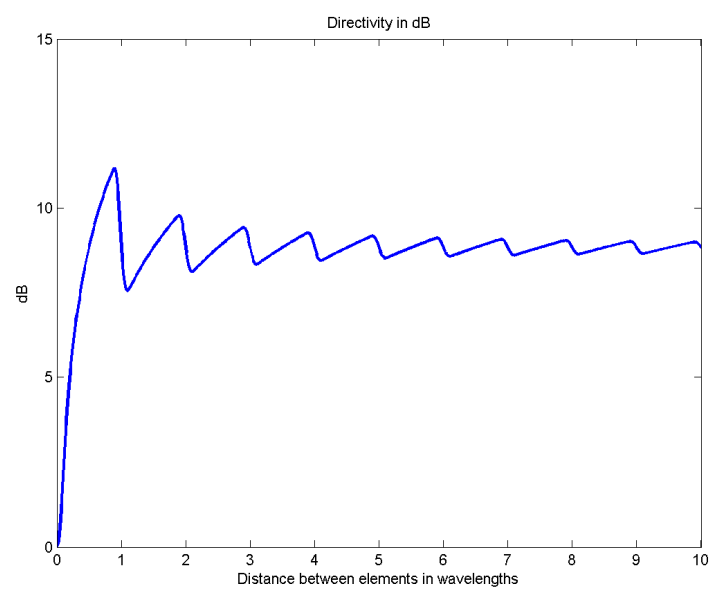

Fig. 5: Efficient computation of the directivity of an 8 element $-20 \mathrm{~dB}$ Chebychev linear array

We notice the expected behaviour in Fig. 6 for a simple linear 8 element array, and notice that in some cases (when the distance is close to 0.9 wavelengths), the directivity of the uniform array might be less than that of a $-20 \mathrm{~dB}$ sidelobe Chebychev array. We can also see that the directivity of the array is not equal to the sum of the element directivity and the isotropic directivity, even if the difference is usually less than $1 \mathrm{~dB}$.

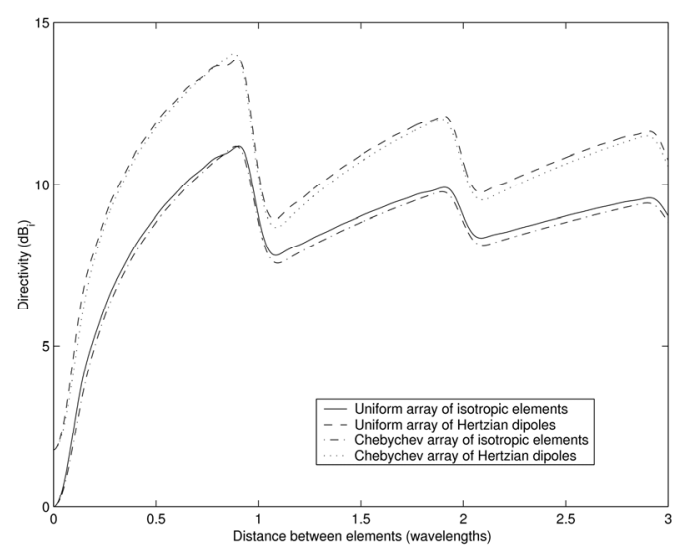

Fig. 6: distance functions for Hertzian dipoles and isotropic elements.

This formula can be further generalised for completely arbitrary patterns, by computing multiple derivatives of the simple sinc function with respect to both $\alpha(\theta)$ and $\beta(\varphi)$.

$$
\left[\operatorname{sinc}\left(\sqrt{\alpha^{2}+\beta^{2}}\right)\right]
$$

\section{APPLICATIONS}

If we are only interested in the far-field of an antenna - true in most cases of indoor wireless computations not too close to the antennas (but those cases guarantee a sufficient signal strength and a sufficient direct to multipath ratio) - the computational time is reduced in proportion to the number of array elements while retaining high accuracy. This is illustrated in one example of a room at ISM frequencies (Fig. 7)

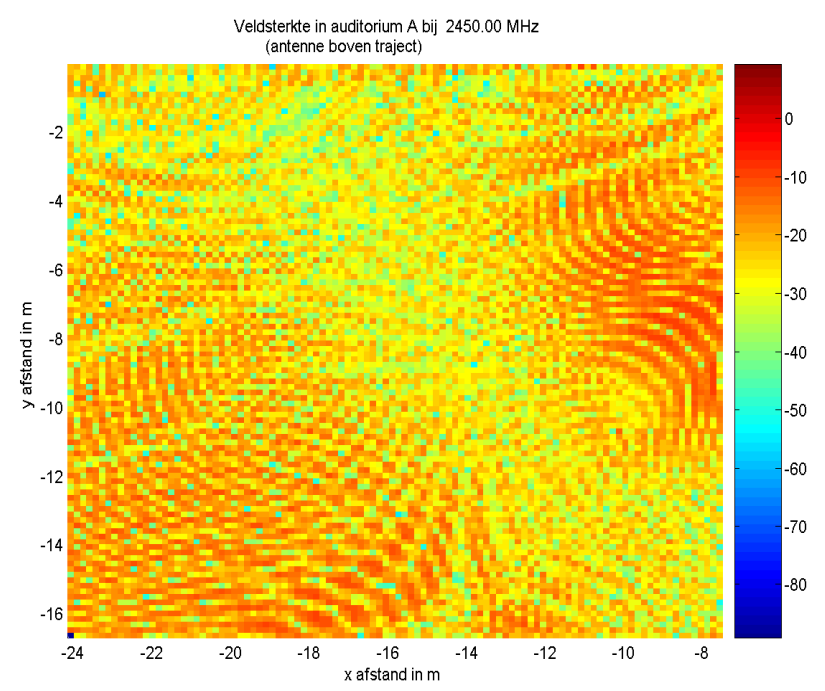

Fig. 7: Field strength in a large auditorium at $2.45 \mathrm{GHz}$.

The difference between a computation with the array factor and the directivity (Fig. 8) and one with the separate elements 
(Fig. 9) is not very large, but the first takes much less computational time (about equal to the number of elements).

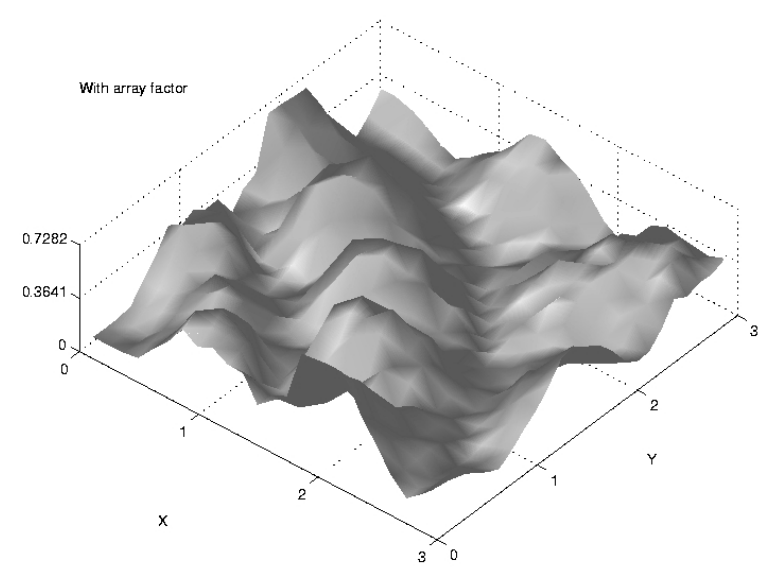

Fig. 8: Field strength in a $3 \times 3 \times 3$ cubiculum at $2.45 \mathrm{GHz}$ with array factor.

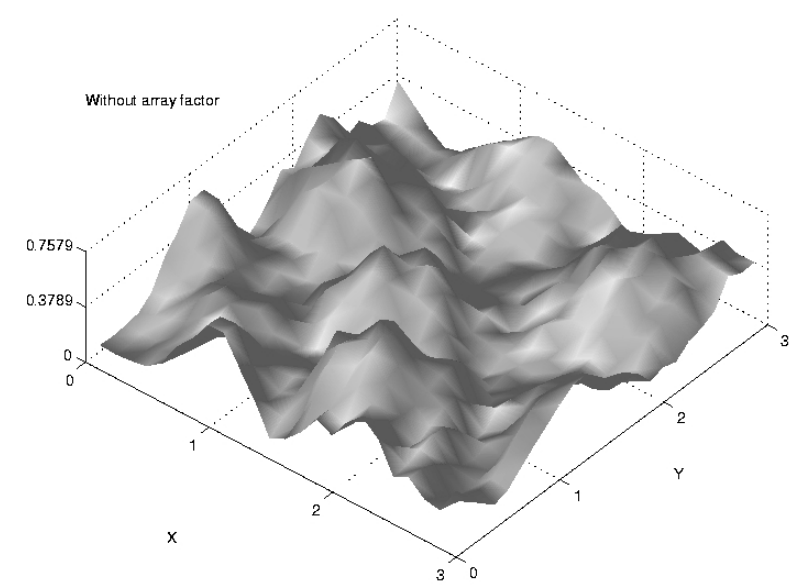

Fig. 9: Field strength in a $3 \times 3 \times 3$ cubiculum at $2.45 \mathrm{GHz}$ with separate elements.

\section{CONCLUSIONS}

Directivities of arbitrary arrays can be easily computed in function of only spherical Bessel functions and the knowledge of the excitation coefficients.

\section{REFERENCES}

[1] W. Stutzmann and G. Thiele, Antenna Theory and Design, John Wiley \& Sons, New York, 1981

[2] M.T. Ma, Theory and Application of Antenna Arrays, Artech House, 1974

[3] I. Gradshteyn and I. Ryzhik, Table of Integrals, Series and Products, (Academic Press, New York, 1980)

[4] I. De Coster, E. Van Lil, "Implementation of Antenna Patterns in EPICS", in Proc. 9th Cost 259 Meeting, Leidschendam, The Netherlands, TD(99)093, 8p., 23-24 September 1999I. S. Jacobs and C. P. Bean, "Fine particles, thin films and exchange anisotropy," in Magnetism, vol. III, G. T. Rado and H. Suhl, Eds. New York: Academic, 1963, pp. 271-350.

[5] E. Van Lil, J.-w. De Bleser, A. Van de Capelle, "Optimal Calculation of the Directivity of Arrays with Azimuthal Element Pattern Symmetry", PIERS2011, Suzhou, China, 12-16 Sepetmber 2011 